\title{
The intraday variability in the radio-selected and X-ray-selected BL Lacertae objects ${ }^{\star}$
}

\author{
J.M. Bai ${ }^{1}$, G.Z. Xie ${ }^{1,2}$, K.H. Li ${ }^{1}$, X. Zhang ${ }^{1,3}$, and W.W. Liu ${ }^{1}$ \\ 1 Yunnan Astronomical Observatory, The Chinese Academy of Sciences, Kunming 650011, China \\ e-mail: gzxie@public.km.yn.cn \\ 2 United Laboratory of Optical Astronomy, The Chinese Academy of Sciences (ULOAC), China \\ 3 Department of physics of Yunnan Normal University, Kunming, China
}

Received September 27, 1997; accepted April 15, 1998

\begin{abstract}
Seven BL Lac objects have been photometrically observed in an effort to study the difference of optical intraday variability between the radio-selected BL Lac objects (RBLs) and X-ray-selected BL Lac objects (XBLs). The objects we observed are selected arbitrarily. They are four RBLs, PKS 0735+178, PKS 0754+101, OJ 287 and BL Lac, and three XBLs, H 0323+022, H 0548-322 and H 2154-304. During the observation all of them exhibited microvariation, and $\mathrm{H} 0323+022$ and H 0548-322 sometimes showed brightness oscillation. PKS $0735+178$ and BL Lac were in their faint states and not very active. It seems that RBLs do not show microvariability more frequently than XBLs.
\end{abstract}

Key words: BL Lacertae objects: general - galaxies: photometry

\section{Introduction}

BL Lac objects are in many ways the most extreme class of active galactic nuclei (AGNs), that have no or only weak emission lines in their spectra and exhibit strong and rapid variability and polarization from radio through $\mathrm{X}$-ray regime. Based on their discovering techniques, they are divided into two subclasses, the radio-selected BL Lac objects discovered by optical spectroscopic identification of radio source, and the X-ray-selected BL Lac objects found by optical spectroscopic identification of candidates from X-ray surveys. Long term monitoring in the optical band shows that XBLs are less variable and polarized than RBLs, the variability amplitude of long term light

Send offprint requests to: J.M. Bai

* Table 2 is only available in electronic form at the CDS via anonymous ftp to cdsarc.u-strasbg.fr (130.79.128.5) curve of XBLs is much smaller than that of RBLs and the percent polarization is lower than that for RBLs (Jannuzi et al. 1994). It is widely believed that BL Lac objects are identical to low-luminosity radio galaxies (FR I, Fanaroff \& Riley 1974) with relativistic jets oriented close to the line of sight. XBLs have the jets orienting at much larger angles to the line of sight than RBLs do (e.g. Stocke et al. 1985; Maraschi et al. 1986; Ghisellini \& Maraschi 1989).

Intraday variability, as defined by Wagner \& Witzel (1995), is used to describe the microvariations on time scales of one day or less. There have been many reports of optical microvariations in BL Lac objects. Wagner and Witzel have given a review for microvariability (Wagner \& Witzel 1995, and reference therein). Heidt \& Wagner (1996) find optical intraday variability in $80 \%$ of the sources in the Stickel's complete sample of radio-selected BL Lac objects (Stickel et al. 1991). If the difference between the XBLs and RBLs is truly caused by the orientation of relativistic jet, the intraday variability in RBLs and XBLs will show some differences. Miller \& Noble (1997) have investigated much smaller microvariability in RBLs and XBLs, and found that RBLs systematically exhibit larger amplitude, more rapid microvariability than XBLs. This is consistent with the RBLs having the jets oriented closer to the line of sight than XBLs.

In this paper, we present the recent observational results from CCD photometric monitoring of seven BL Lac objects. As a preliminary discussion of these data, we have compared the difference in intraday variability between radio-selected and X-ray-selected BL Lac objects.

\section{Observation and data reduction}

The observation presented here were carried out with the one-meter telescope of Yunnan Observatory during November 1996 to January 1997. The telescope is 
equipped with a direct CCD $(1024 \times 1024$ pixels $)$ camera at the Cassergrain focus. The CCD was bought from Princeton Instruments Company, U.S.A. The field is about $6.5^{\prime} \times 6.5^{\prime}$. The filters are standard Johnson broadband filters as:

$$
\begin{aligned}
& B-\operatorname{GG} 385(2 \mathrm{~mm})+\operatorname{BG} 12(1 \mathrm{~mm})+\operatorname{BG} 18(2 \mathrm{~mm}) \\
& V-\operatorname{GG} 495(2 \mathrm{~mm})+\operatorname{BG} 18(2 \mathrm{~mm}) \\
& R-\operatorname{RG} 610(3 \mathrm{~mm})+66.2500(1 \mathrm{~mm}) \\
& I-\operatorname{RG} 715(3 \mathrm{~mm})+60.5050(1 \mathrm{~mm}) .
\end{aligned}
$$

The exposure time is $300-400$ seconds for $B$ and $V$ filters, $100-200$ seconds for $R$ and $I$ filters. The sources we observed are listed in Table 1. They are selected arbitrarily. The standard stars in the field are taken from Smith et al. $(1985,1991)$ (see Table 1).

The results are given in Table 2. The first column is the observing date, the second the Julian date, the third the magnitude, the fourth the observational $1 \sigma$ uncertainty level and the last the filter used. All observing data were processed using the photometric tool, APPHOT, in IRAF software package. The flat field images were taken at dusk and dawn when possible. The bias (taken at least $15 \mathrm{im}-$ ages each night) were taken at the beginning of the observation and the end. The source magnitude is given as the average of those derived with respect to the two brightest standard stars in the image frame containing the source which present the smallest variations in their differential magnitudes. The errors quoted in Table 2 are calculated from the two standard stars, star 1 and star 2 , in the usual way,

$\sigma=\sqrt{\frac{\Sigma \delta_{i}}{N-1}} \quad i=1,2, \ldots N$,

where $\delta_{i}=\left(m_{2}-m_{1}\right)_{i}-\overline{m_{2}-m_{1}}, m_{2}-m_{1}$ is the differential magnitude between star 2 and star $1, \overline{m_{2}-m_{1}}$ is the mean differential magnitude, and $\mathrm{N}$ is the number of the observations. Star 1, which is the brightest standard star, is also used for calibration, and star 2 is standard star whose brightness is comparable with the source or a little fainter than the source. When all the standard stars are brighter than the source, the actual observational error for the source is greater than the $\sigma$ calculated as above, and the errors have been given according to the typical uncertainties for sources of brightness comparable with the source.

\section{Observational results}

The observation have been done in four $B, V, R, I$ optical bands. The light curves complement each other, since in some cases we have observations in one band, but not on the others. In the following description for the variability behaviour of each individual object, we will combine the data in all four bands. Since the $B$ band light curves are often poor in time resolution and accuracy, the data in the $B$ band are paid less attention than the data in the other three bands.

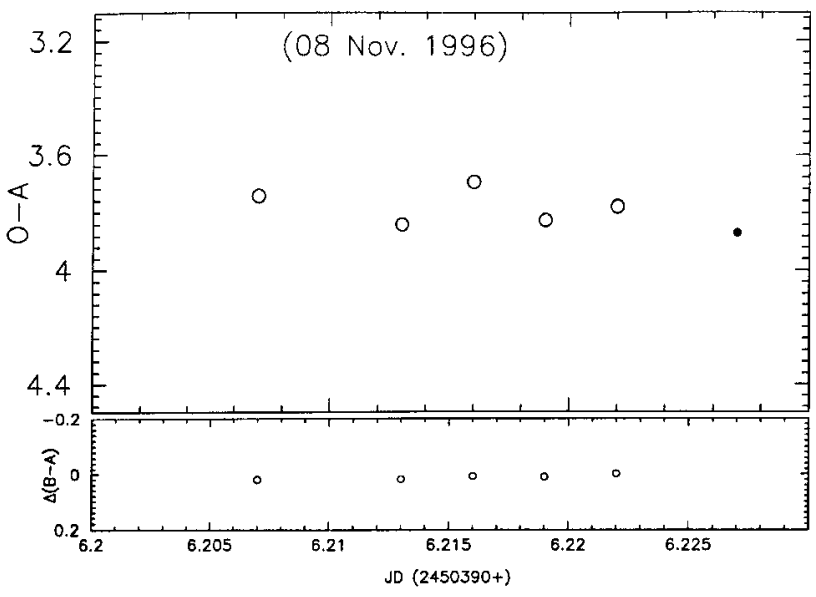

Fig. 1. Light curve of H $0323+022$ on 08 November 1996. Star $A$ and $B$ are of the sequence of Smith et al. (1991)

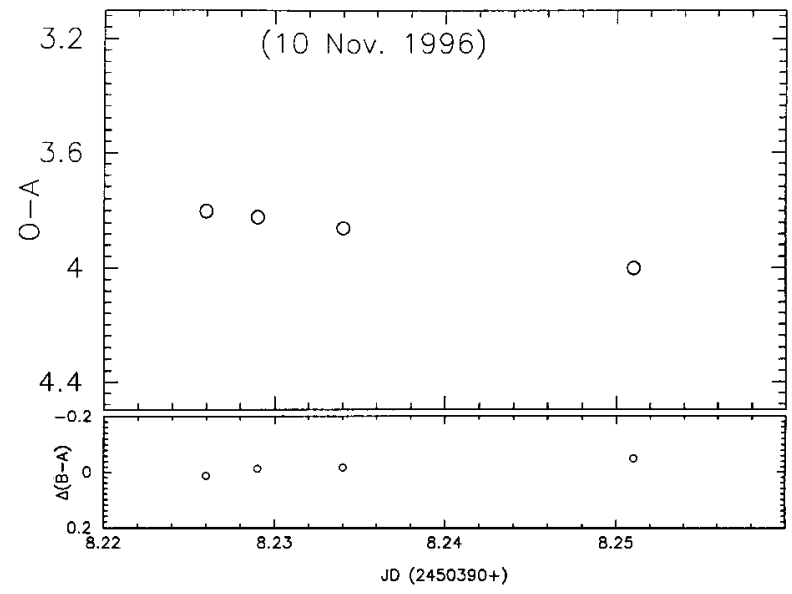

Fig. 2. Light curve of H $0323+022$ on 11 November 1996

\section{1. $H 0323+022$}

$\mathrm{H} 0323+022$ is a bright X-ray-selected BL Lac object. Variability on time scale of hours have been observed in the X-ray regime (Margon \& Jacoby 1984). During the three years monitoring (1987-1990), its range of variation is $16.13-17.07 \mathrm{mag}$ in the $V$ band (Jannuzi et al. 1993).

The light curves of our observations are displayed in Figs. 1-5. The top panels display the differential magnitudes between $\mathrm{H} 0323+022$ and the comparison star $A$, OA. Star $A$ and $B$ are of the sequence of Smith et al. (1991). The bottom panels display the deviations of differential magnitudes from the mean differential magnitude between comparison star $B$ and $A, \triangle(B-A)$, which provide an indication of the stability of the standard stars used for calibration and in the case that $\mathrm{O}$ has a comparable magnitude with $B$ (like BL Lac and OJ 287, there are more than two standard stars in its field for selection), provide a reliable measure of the observational uncertainty. The open circles (०) are for $I$ band data, filled circles $(\bullet)$ are 
Table 1. The list of the sources

\begin{tabular}{llcccl}
\hline Object & name & RA $(1950.0)$ & Dec(1950.0) & selection & standard stars \\
\hline $0323+022$ & H & $03: 23: 38$ & $02: 14: 47$ & X & Smith et al. (1991) \\
$0548-322$ & PKS, H & $05: 48: 49$ & $-32: 17: 07$ & X & Smith et al. (1991) \\
$0735+178$ & PKS & $07: 35: 14$ & $17: 49: 09$ & R & Smith et al. (1985) \\
$0754+101$ & PKS & $07: 54: 23$ & $10: 04: 39$ & R & Smith et al. (1985) \\
$0851+102$ & OJ 287 & $08: 51: 57$ & $20: 17: 59$ & R & Smith et al. (1985) \\
$2200+420$ & BL Lac & $22: 00: 39$ & $42: 02: 08$ & R & Smith et al. (1985) \\
$2155-304$ & PKS, H & $21: 55: 58$ & $-30: 27: 55$ & X & Smith et al. (1991) \\
\hline
\end{tabular}

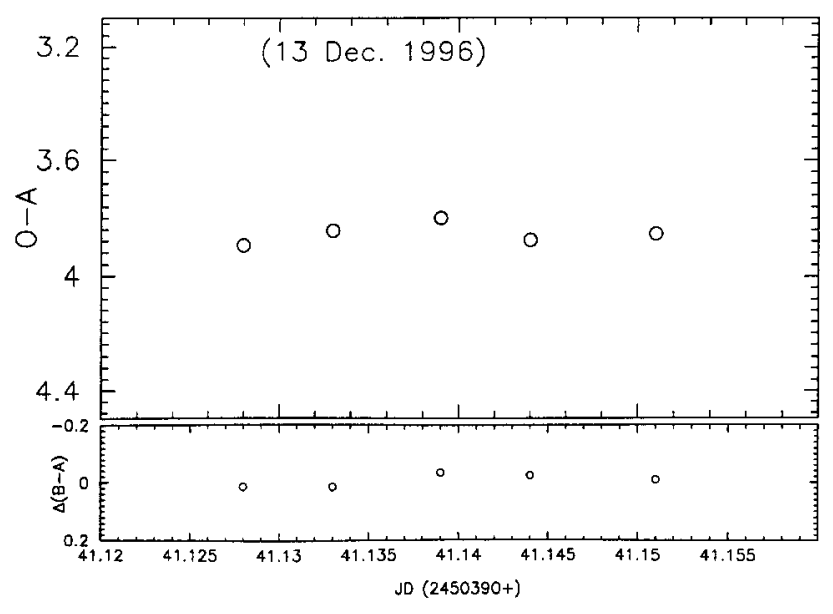

Fig. 3. Light curve of H $0323+022$ on 13 December 1996

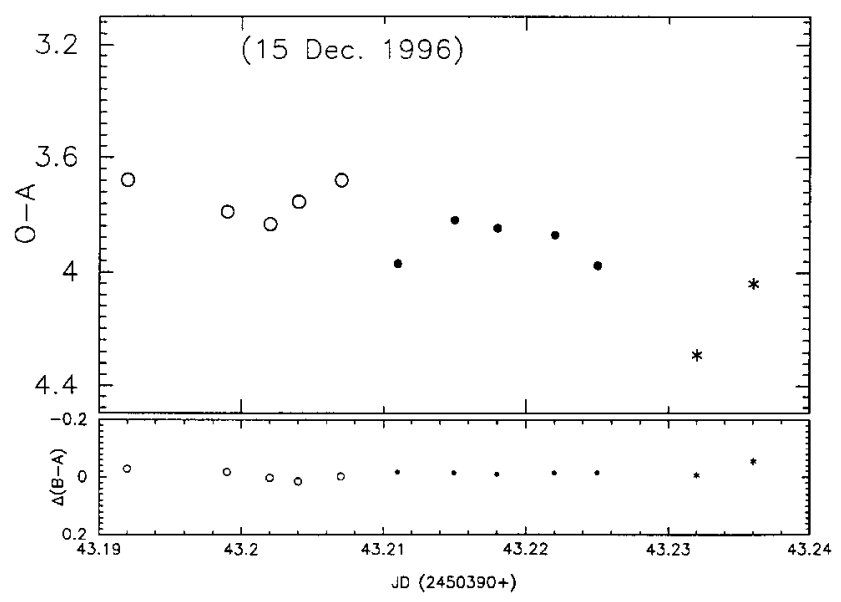

Fig. 4. Light curve of H $0323+022$ on 15 December 1996

for $R,(*)$ for $V$ and $(\oplus)$ for $B$. All subsequent figures will be constructed in the same fashion. Our observations obtained on 08 November 1996 show that the source exhibited no convincing microvariation (Fig. 1). Observations obtained on 10 November indicate a gradual decline in the source's brightness of $\triangle I=0.23 \mathrm{mag}$ (Fig. 2). The source was next observed on 13 december 1996, and showed the same brightness level as that in November. No convincing microvariation in the source was recorded during this night (Fig. 3). Figure 4 shows the observation obtained

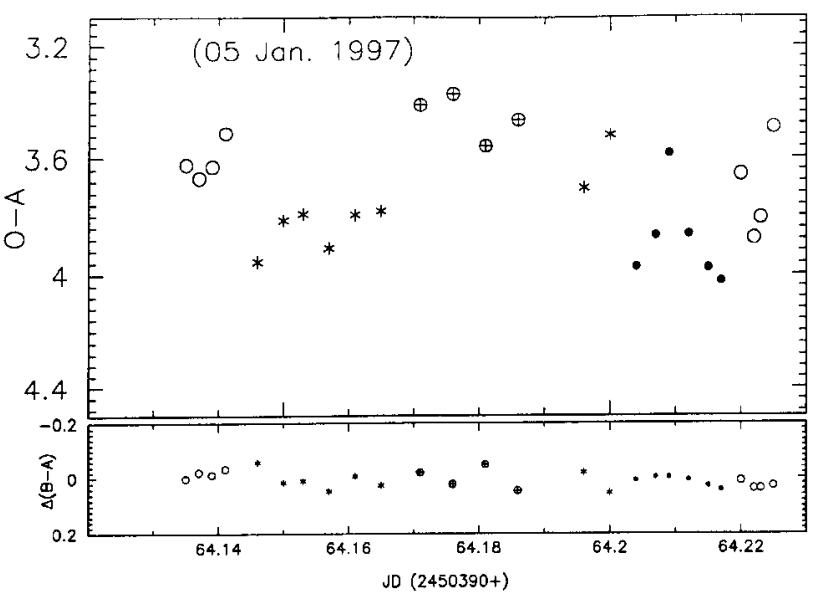

Fig. 5. Light curve of H $0323+022$ on 05 January 1997

on 15 December 1996. The source first declined by $\triangle I=$ $0.14 \mathrm{mag}$ in 15 minutes (JD $=2450433.192$ to 2450433.202 ), then began to brighten continuously. From the subsequent $R$ band observations, we can see that the source continued this rise in brightness till JD = 2450433.215, lasting 18 minutes, then began to fade till JD $=2450433.232$ and began another rising. The amplitude of this flare is at least $0.30 \mathrm{mag}$ in the $I$ band (take $R-I=16.31-15.50$ ). The observations obtained on 05 January 1997 are displayed in Fig. 5. From JD $=2450450.137$ to 2450450.141 the source brightened by $\triangle I=0.15 \mathrm{mag}$ in about five minutes. In the subsequent $V, B$ band observation it continued this rise till $\mathrm{JD}=2450454.176$, then faded by $\triangle B=$ $0.22 \mathrm{mag}$. On JD $=2450451.181$ the source brightened again by $\triangle V=0.18 \mathrm{mag}$ in five minutes then $\triangle R=$ 0.38 mag in 8 minutes, then quickly faded by $\triangle R=$ $0.42 \mathrm{mag}$ in 12 minutes, $\Delta I=0.21 \mathrm{mag}$ in about 2 minutes, and again began to brighten. The amplitude of this flicker is about $\triangle R=0.63 \mathrm{mag}$ (take $R-I=$ $16.33-15.47)$.

\section{2. $H 0548-322$}

H $0548-322$ is a X-ray-selected BL Lac object. During their 3-year monitoring, Jannuzi et al. (1993) found this object to vary by more than $0.5 \mathrm{mag}$. H $0548-322$ has 


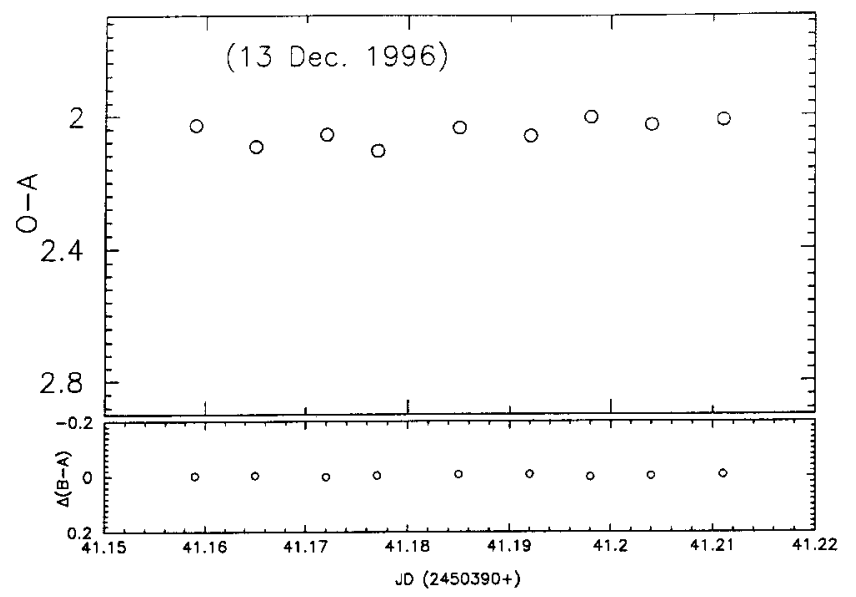

Fig. 6. Light curve of H 0528 - 322 on 13 December 1996. Star $A$ and $B$ are of the sequence of Smith et al. (1991)

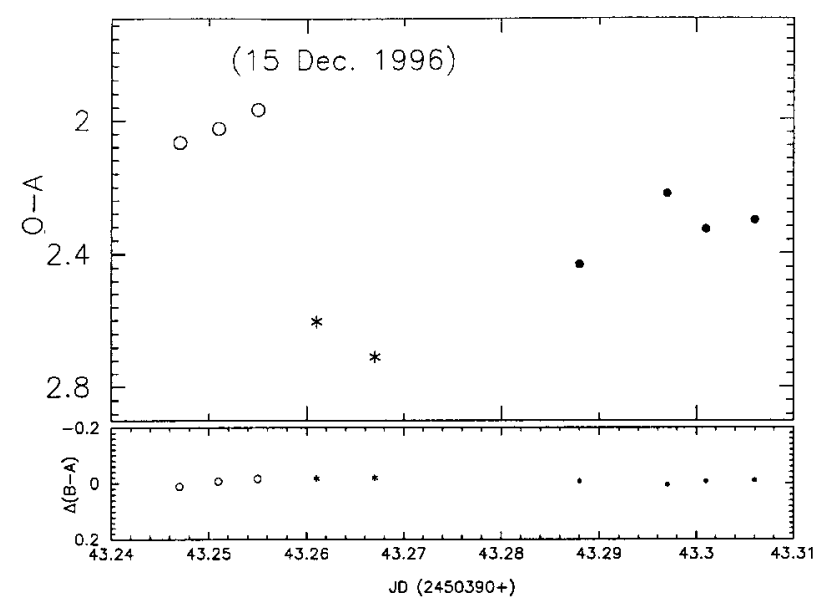

Fig. 7. Light curve of H 0528 - 322 on 15 december 1996

been included in our monitoring program since 1992, and a flare of $\triangle V=0.58 \mathrm{mag}$ in 67 minutes was detected on 28 February 1992 (Xie et al. 1995).

Figure 6 displays our new observations obtained on 13 December 1996. The light curve indicates that the source was stable on this night. On the next night the source was observed brightening $0.08 \mathrm{mag}$ in the $I$ band within about 11 minutes (from JD $=2450433.247$ to 2450433.255 ). At the end of the observation another flicker was detected. Within 13 minutes (JD $=2450433.288$ to 2450433.297 ) the source brightened by $0.21 \mathrm{mag}$ in the $R$ band, then faded $\sim 0.08 \mathrm{mag}$ to the end of our observation (see Fig. 7 ). H 0548-322 was next observed on the night of 01 January 1997. On JD $=2450450.155$ the source began to oscillate in brightness, and reached local maxima on JD = $2450450.172,2450450.192$ and 2450450.228 , with an amplitude being about $0.1 \mathrm{mag}$ in the $R$ band (Fig. 8). The observations obtained on 3 January 1997 are displayed in Fig. 9. From JD $=2450452.120$ to 2450452.137 the source faded $\triangle I=0.1 \mathrm{mag}$, then brightened by $\triangle I=$ $0.08 \mathrm{mag}$ (to JD $=2450452.157$ ). From JD $=2450452.174$

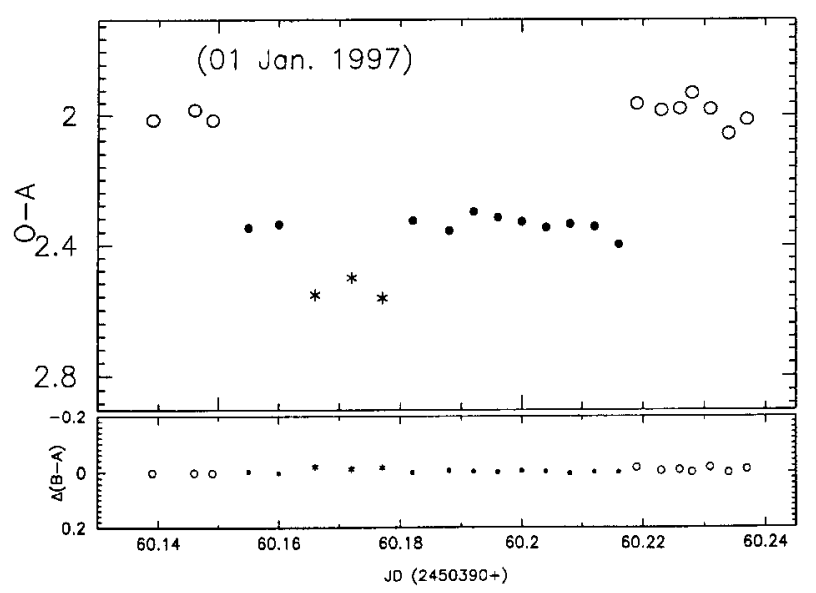

Fig. 8. Light curve of H 0528 - 322 on 01 January 1997

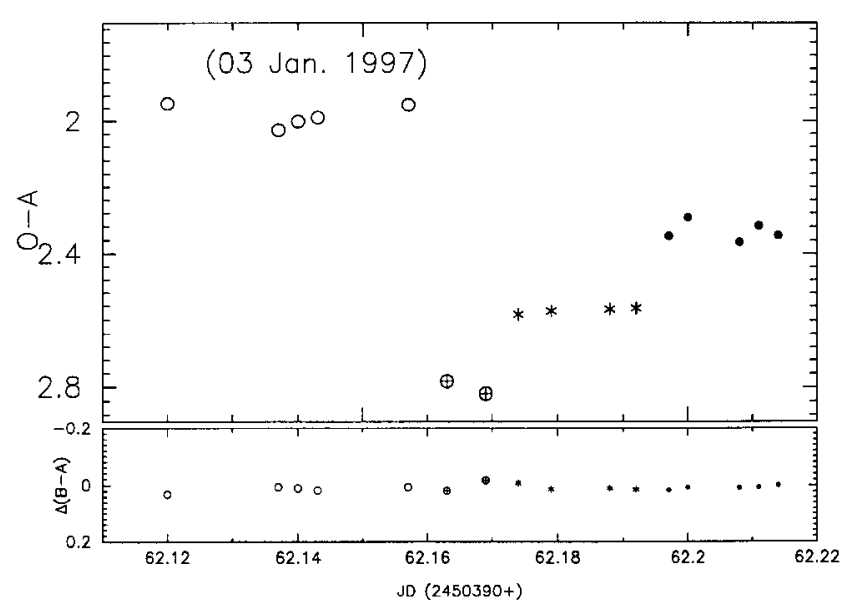

Fig. 9. Light curve of H 0528 - 322 on 03 January 1997

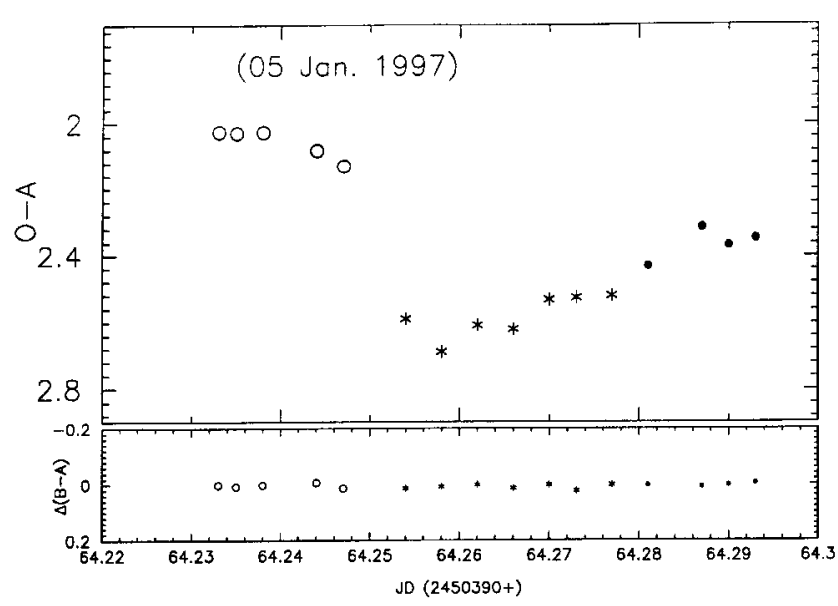

Fig. 10. Light curve of H 0528 - 322 on 05 January 1997 


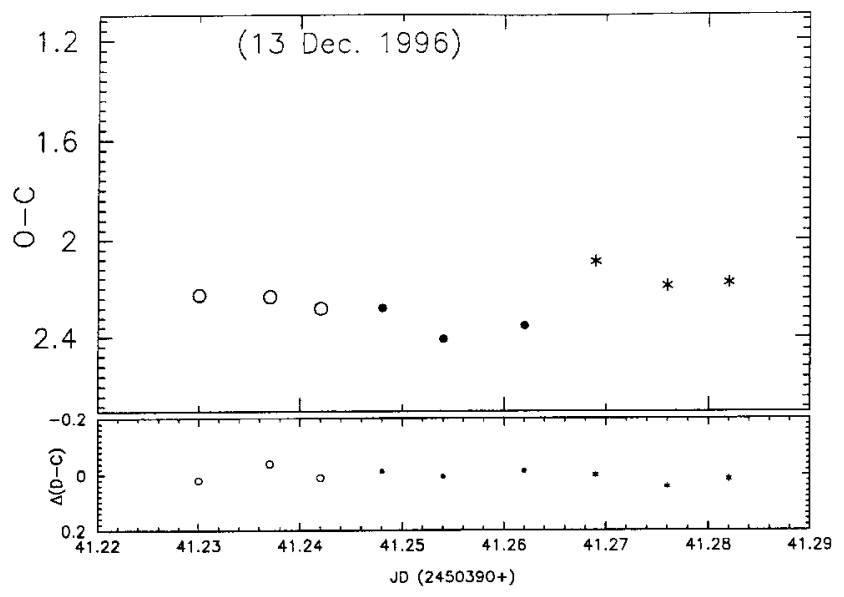

Fig. 11. Light curve of PKS 0735+178 on 13 December 1996. Star C and D are of the sequence of Smith et al. (1985)

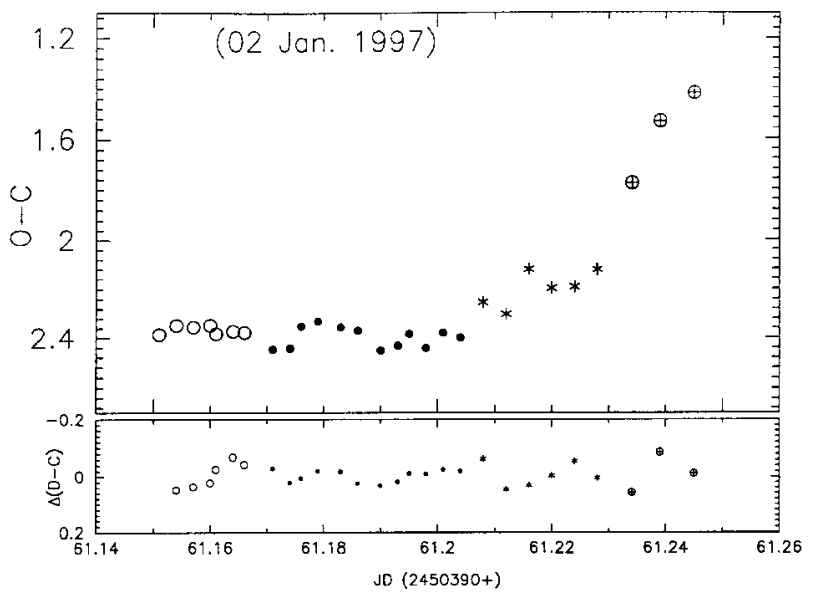

Fig. 12. Light curve of PKS 0735+178 on 02 January 1997

to 2450452.208 the source brightened by $\triangle R=0.08 \mathrm{mag}$ (take $V-R=15.92-15.38$ ), then began to fade to the end of our observation. On 5 January 1997, the source first gradually faded $\triangle I \sim 0.19 \mathrm{mag}$ (take $V-I=15.92-$ 15.38) within about 36 minutes (from $\mathrm{JD}=2450433.233$ to 2450454.258 ), then gradually brightened by $\triangle V \sim$ 0.28 mag (take $V-R=15.88-15.47$ ) in about 38 minutes (from JD $=2450433.258$ to 2450454.287 , see Fig. 10). In general, the source exhibited no long term variation from December 1996 to January 1997 (see Figs. 6-10). It kept at a brightness level of $V \approx 15.9 \mathrm{mag}, R \approx 15.3 \mathrm{mag}$ and $I \approx 14.7$ mag.

\section{3. $P K S 0735+178$}

This is a radio-selected BL Lac object. Historical light curves indicate that its range of variability in the $B$ band is 13.90 - $17.72 \mathrm{mag}$ (Pollock et al. 1979; Xie 1987, 1988b, 1990, 1991, 1994). Figure 11 shows the new observations obtained on 13 December 1996. During the observation of 75 minutes, the source faded steadily at least by $\triangle I=$

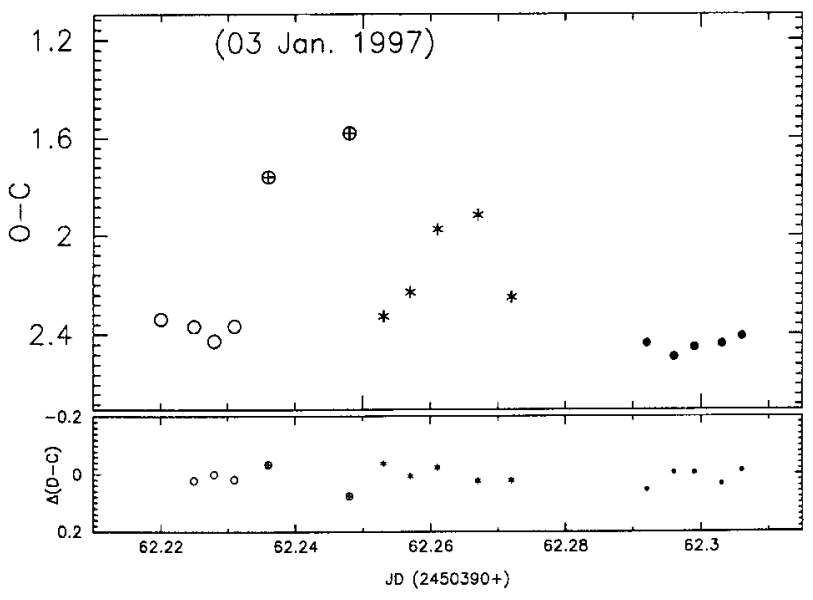

Fig. 13. Light curve of PKS 0735+178 on 03 January 1997

0.22 mag (take $R-I=16.13-15.63, V-R=$ $16.49-16.21)$. The observations obtained on 02 January 1997 are displayed in Fig. 12. During the first 19 minutes $I$ band observation, the source was stable, then on $\mathrm{JD}=2450451.171$ it began to brighten and on JD = 2450451.179 reached a local maximum of $R=16.19 \mathrm{mag}$, then began to fade. On JD $=2450451.190$ it reached a local minimum of $R=16.29 \mathrm{mag}$, and again began to brighten slowly by about 0.52 mag (take $V-R=$ $16.69-16.26, B-V=16.53-17.13)$ to the end of the observation. However, the standard stars are not very stable (see the bottom panel in Fig. 12). On 3 January 1997, we recorded a rapid flare for this source. From $\mathrm{JD}=2450452.228$ to 2450452.267 (in 56 minutes) it brightened by about $0.65 \mathrm{mag}$ (take $B-I=17.16-15.71$, $B-V=16.92-16.63)$, then fade sharply about $0.41 \mathrm{mag}$ (take $V-R=16.64-16.26$, see Fig. 13). Figures 11-13 show no long term variation trends, the source kept at a faint level of $B \approx 17.0 \mathrm{mag}, V \approx 16.6 \mathrm{mag}$.

\subsection{PKS $0754+101$}

PKS $0754+101$ is a radio selected BL Lac object. The near-IR observations by Falomo et al. (1992) indicated that this source was not very active from 1987 to 1989 . Massaro (1995) found that the range of variability was about one magnitude during their near-IR observation from 1986 to 1992.

Our observations are displayed in Figs. 14-16. On the two successive nights of 13 and 14 December 1996, the source exhibited no intranight variation and no microvariations each night (Figs. 14 and 15). Two weeks later, on 2 Jan. 1997 the source's brightness was 0.4 mag fainter in the $V$ band. First the source brightened by $0.17 \mathrm{mag}$ in the $I$ band within about 11 minutes (from JD $=2450451.257$ to 2450451.265). At the end of the observation on this night, the source exhibited a quick decrease in its brightness of $\triangle B=0.47 \mathrm{mag}$ in 22 minutes (Fig. 17). 


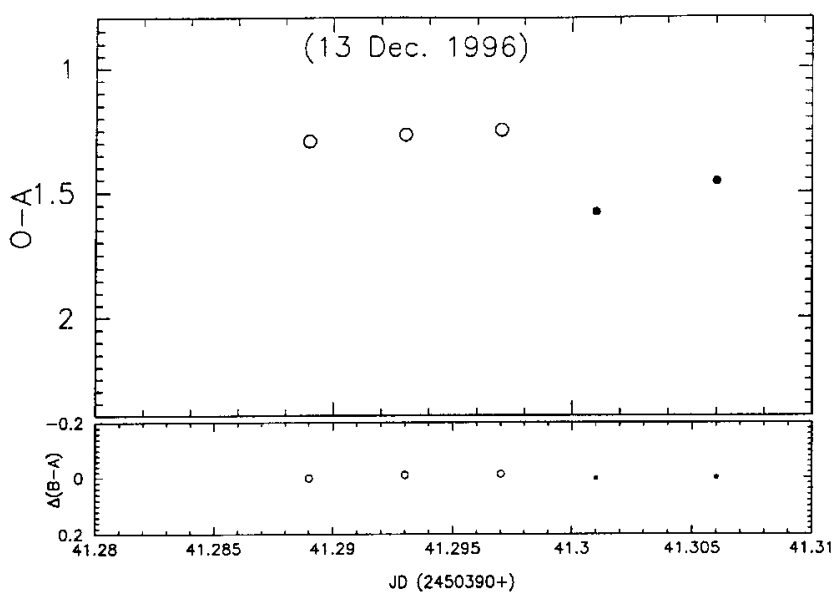

Fig. 14. Light curve of PKS 0754+101 on 13 December 1996. Star $A$ and $B$ are of the sequence of Smith et al. (1985)

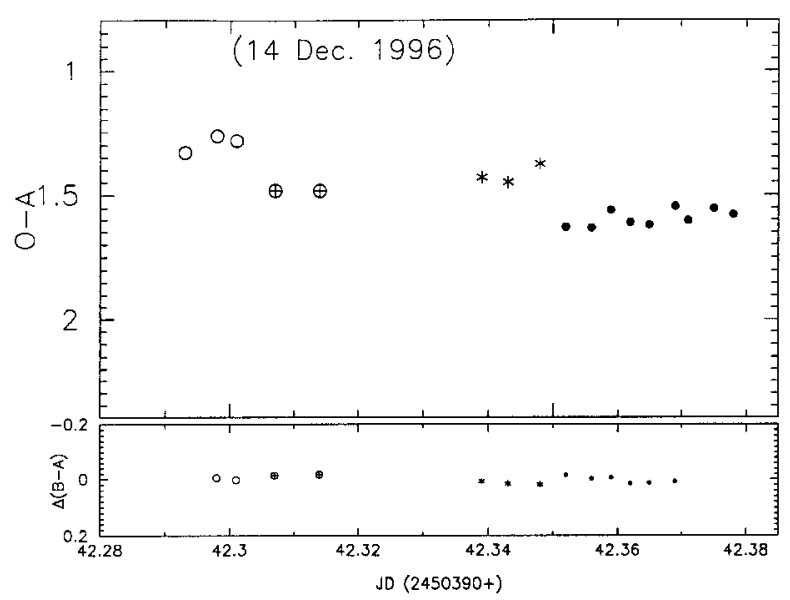

Fig. 15. Light curve of PKS 0754+101 on 14 December 1997

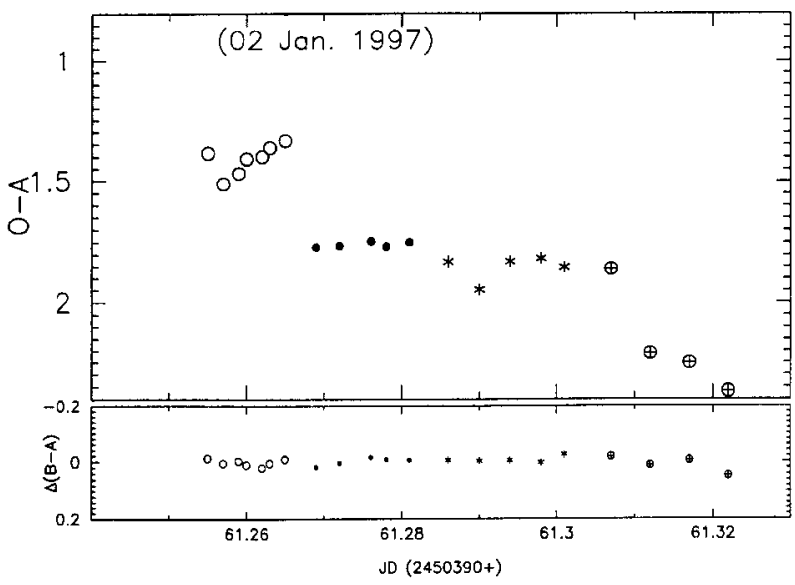

Fig. 16. Light curve of PKS 0754+101 on 02 January 1997

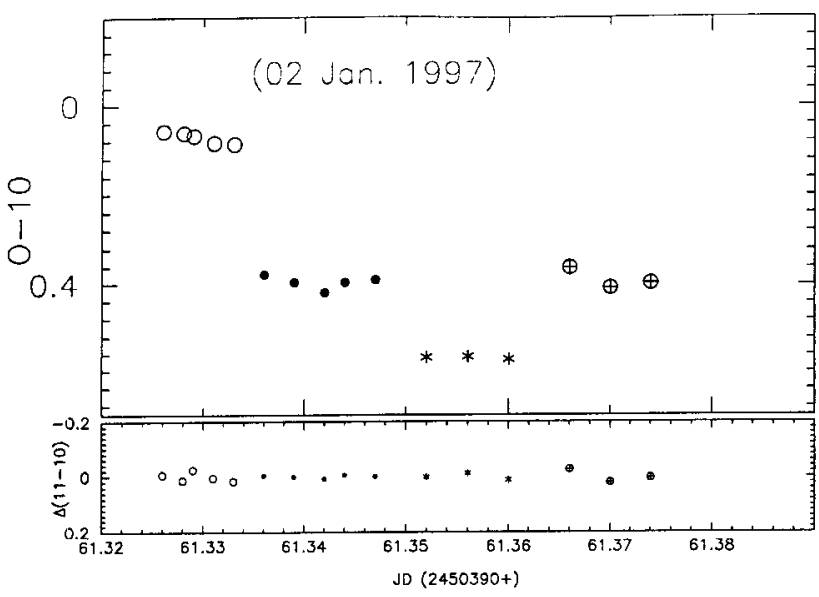

Fig. 17. Light curve of OJ 287 on 02 January 1997. Star 10 and 11 are of the sequence of Smith et al. (1985)

\subsection{OJ 287}

OJ 287 is a bright and classical BL Lac object. There are a lot of information on it in the literature. It has been included in almost all optical and radio monitoring campaigns conducted on blazars (Takalo 1994). The historical light curves have been compiled by Craine \& Warner (1973); Visvanathan \& Elliot (1973); Miller et al. (1976); Gadia \& Roser (1982); Webb et al. (1988); Sillanpää et al. (1988); Kidger et al. (1992); Takalo (1994). Takalo (1994) has collected all the available observations for this source published by the end of 1992, and found that the total range of variability observed for this object was 18.0 - $12.5 \mathrm{mag}$ in the $B$ band, more than 5 mag. Based on the historical light curves dating back to as early 1894, Sillanpää et al. (1988, 1996a) and Kidger et al. (1992) obtained a variability period of 11.65 years and proposed that OJ 287 is a binary pair of supermassive black holes with an orbital period of 9 years in the rest frame of OJ 287. The observations during 1993-1996 confirmed this discovery (Kidger et al. 1995; Sillanpää et al. 1996a,b). Observations also indicate that the constructed light curves seem to show indication at some shorter periods. Kinman et al. (1974) found an 8-day period for its variability, and Xie et al. (1994) confirmed this periodicity. In addition to long-term variations, this objects has also been observed to exhibit variations with time scales of a day or less.

OJ 287 has been included in our monitoring program since the early 1980's. However during this observing season, OJ 287 was only observed on one night, 2 January 1997. The observations are displayed in Fig. 17. From $\mathrm{JD}=2450451.326$ to 2450451.340 , the source underwent a continuous decline in 22 minutes, then began to brighten. The brightness in the $B$ band on this night was about $B=15.5 \mathrm{mag}$, one magnitude brighter than the mean level of $16.5 \mathrm{mag}$ since 1985 (Takalo 1994), and $1.1 \mathrm{mag}$ fainter than the maximum of $B=14.4 \mathrm{mag}$ in November 


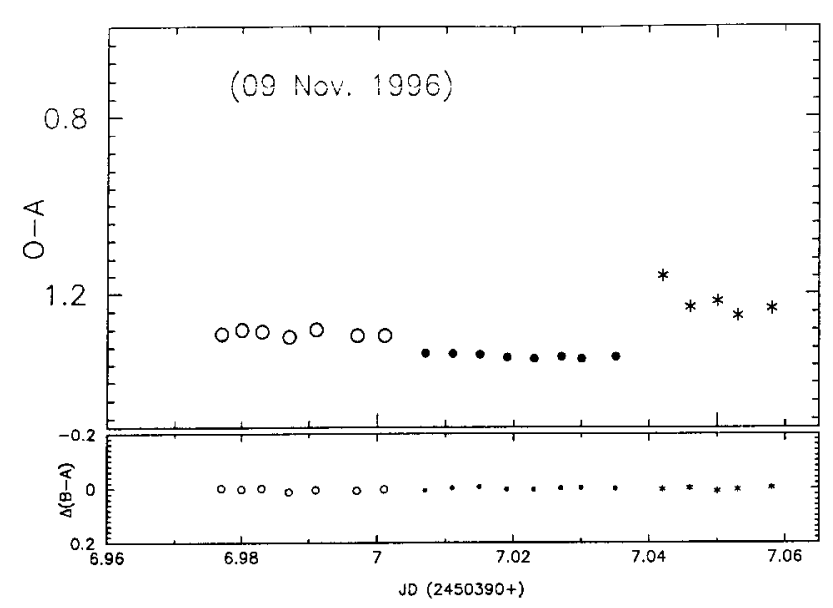

Fig. 18. Light curve of H 2154- 304 on 09 November 1996. Star 2 and 3 are of the sequence of Smith et al. (1991)

1994, which shows that OJ 287 was still in its high state since the outburst in 1993/94.

\section{6. $H 2154-304$ (PKS $2155-304)$}

X-ray-selected, H $2154-304$ is a well observed BL Lac object. The earliest optical monitoring for it by Miller \& McAllster (1983) revealed that the source was not very violent in the optical regime. Pica et al. (1988) have reported that the range of variation of $\mathrm{H} 2154-304$ was 12.93 - $14.00 \mathrm{mag}$ in the $B$ band during five years (19791986). Carini \& Miller (1992a) have presented their longterm photometric monitoring results (1979-1990) with a range of variation of $12.33-13.86$ in the $V$ band. Zhang \& Xie (1996) have constructed an historical light curve for $\mathrm{H} 2154-304$ in which the variation ranges were $12.82-$ $14.20 \mathrm{mag}$ in the $B$ band. H 2154-304 is well known for its short time scales of variability from optical to X-ray wavelengths (Brindle 1986). Large amplitude X-ray flux variations on a time scale of a few hours was found during the EXOSAT observations in 1983 and 1985 (Giommi et al. 1990). Optical variation of the order of one day have been observed by Smith et al. (1992). A world wide campaign to monitor $\mathrm{H} 2154-304$ had been undertaken throughout 1991 November, in which H 2154 - 304 were simultaneously observed in X-ray, ultraviolet, optical, infrared and radio regime, and dramatic, large-amplitude rapid oscillations of about one day have been observed (Urry 1993; Brinkman 1994; Courvoisier 1995).

H 2154-304 has been included in our monitoring program since 1993, and the results show that the object was found with a 0.5 mag flare (Xie et al. 1995). We carried out new observations during 09-11 November 1996. The observations indicate that the source was stable and faint at the level of $V=13.26 \mathrm{mag}, 0.6 \mathrm{mag}$ brighter than the faintest magnitude of 13.86 .

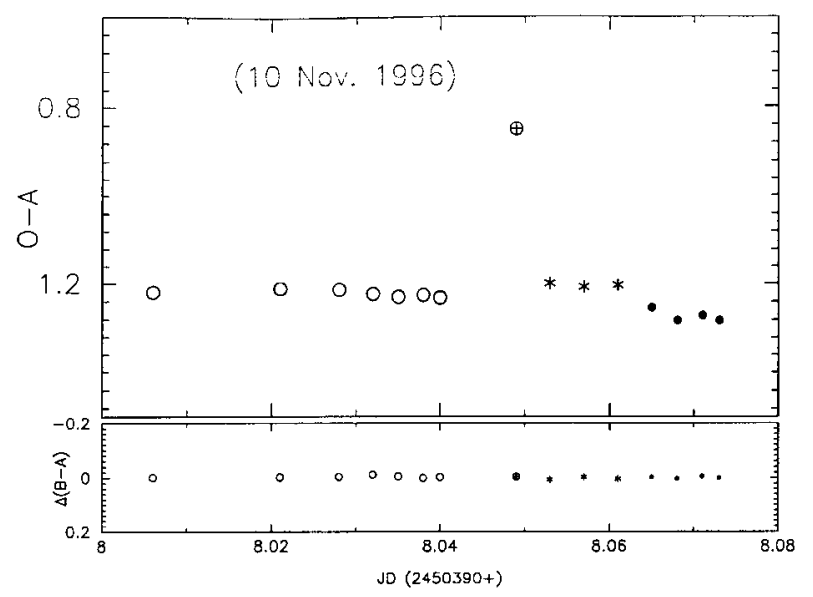

Fig. 19. Light curve of H 2154 - 304 on 10 November 1996

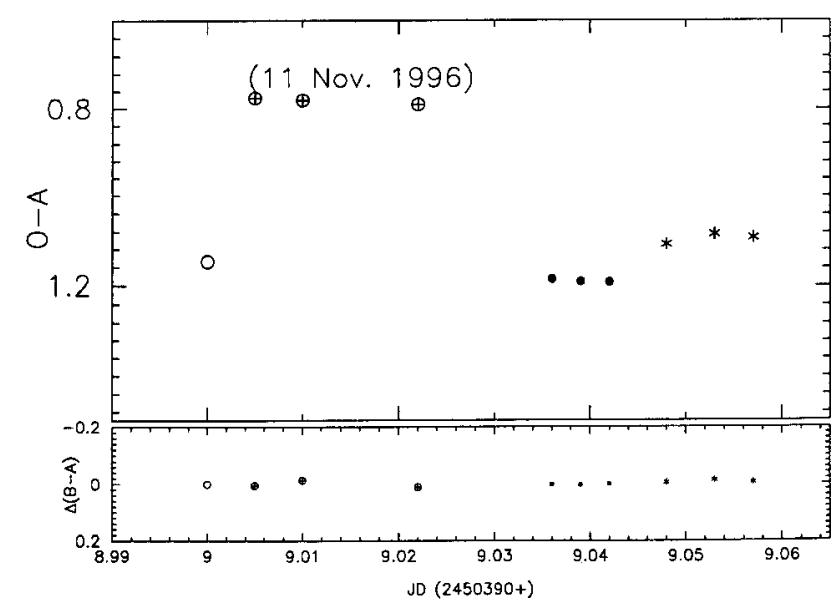

Fig. 20. Light curve of H 2154 - 304 on 11 November 1996

The observations obtained on 9 November 1996 are displayed in Fig. 18. The source was stable during the first 82 minutes. At the end of our observation the source exhibited a slow decrease in its brightness of $\triangle V=$ $0.09 \mathrm{mag}$ (see Fig. 18). On the next night, 10 November the source was about 0.1 mag brighter, and also showed a slow decrease (Fig. 19). On 11 November 1996 the source was stable, but was about 0.1 mag brighter than it was on the night before (Fig. 20).

\section{7. $B L L a c$}

BL Lac is the prototype of the BL Lac object class of AGN and one of the best studied blazars. Shen \& Usher (1970) have investigated its historical light curves, and found a range of variation of 4.2 magnitudes in the $V$ band and a strong outburst in which BL Lac varied over nearly its entire range $(12.4 \leq B \leq 16.7 \mathrm{mag})$ during 400 days. The most recent investigations on its long term variability has been done by Webb et al. (1988) and Carini et al. (1992b) which shows that the faintest magnitude is $B=17.99 \mathrm{mag}$ and $V=16.73 \mathrm{mag}$. 


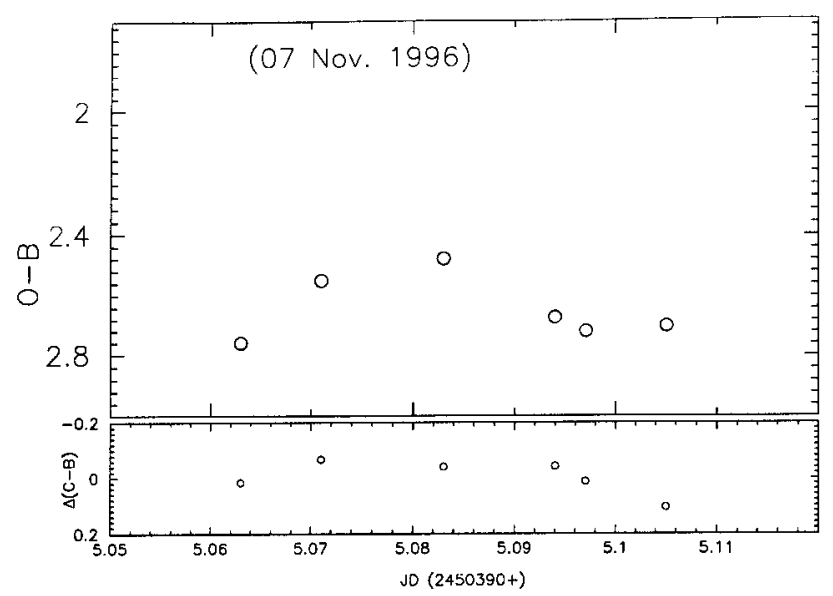

Fig. 21. Light curve of BL Lac on 07 November 1996. Star B and $\mathrm{C}$ are of the sequence of Smith et al. (1985)

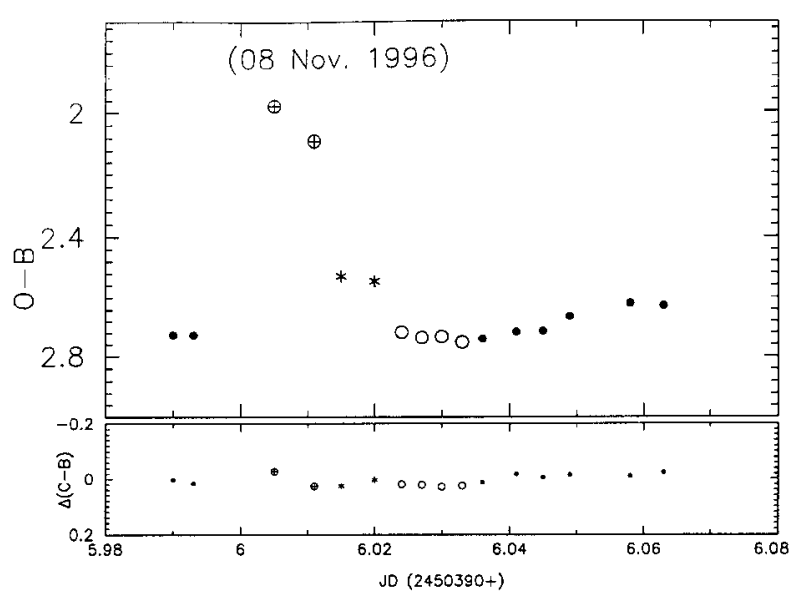

Fig. 22. Light curve of BL Lac on 08 November 1996

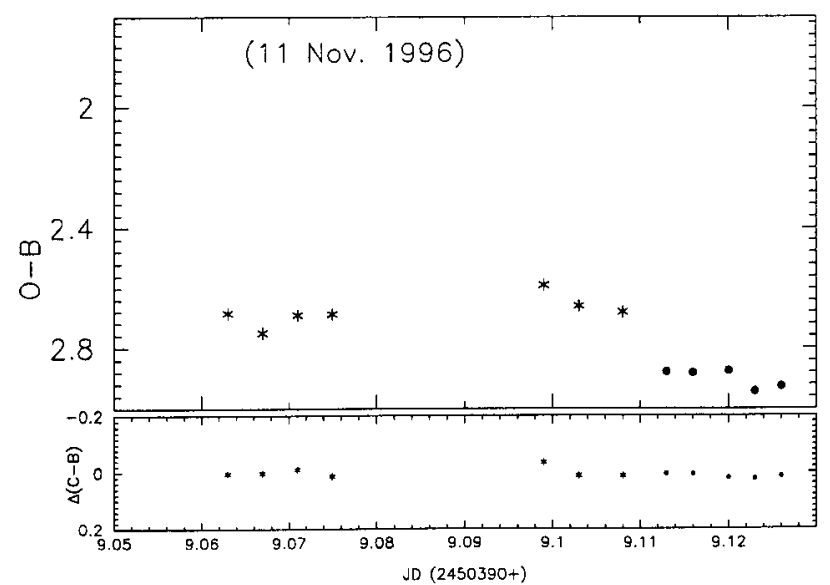

Fig. 23. Light curve of BL Lac on 11 November 1996

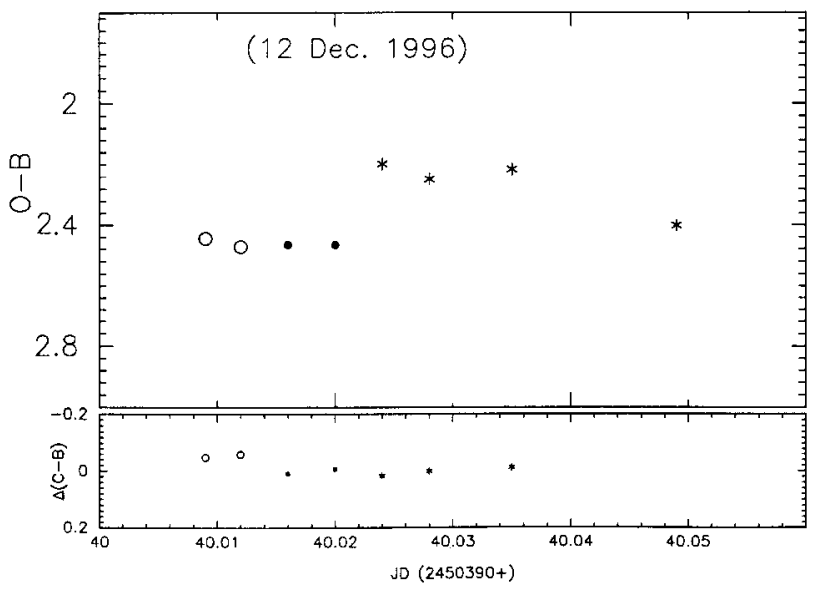

Fig. 24. Light curve of BL Lac on 12 December 1996

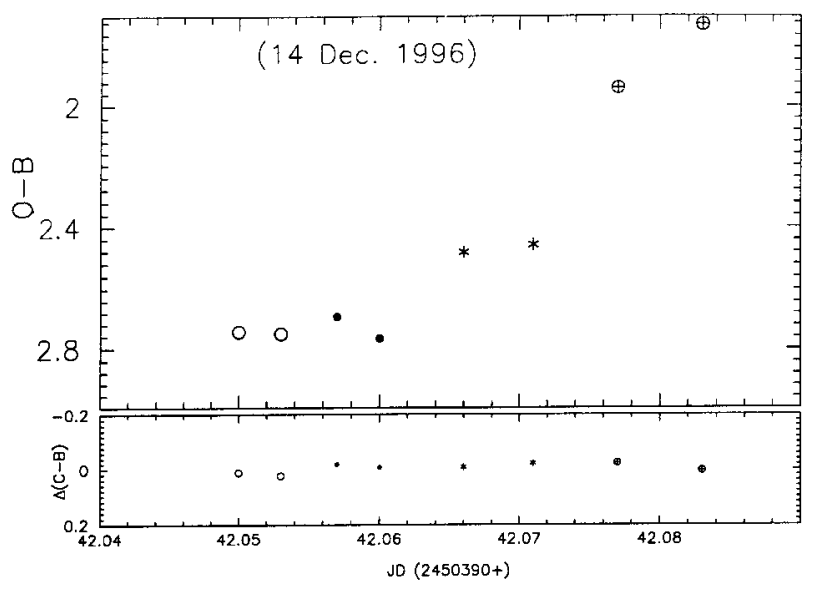

Fig. 25. Light curve of BL Lac on 14 December 1996

BL Lac has been monitored since the beginning of our monitoring program in 1980. A burst of $0.56 \mathrm{mag} / 40 \mathrm{~min}$ in the $B$ band was found in 1985 and confirmed in 1988 (Xie et al. 1988, 1990). A burst of $0.48 \mathrm{mag} / 2 \mathrm{hr}$ in the $B$ band was observed on 1 October 1991, and another burst of $0.37 \mathrm{mag} / 20 \mathrm{~min}$ in the $B$ band was observed on 3 November 1991 (Xie et al. 1994). Figure 21 shows the new observations obtained on 07 November 1996. The light curve shows that BL Lac brightened $\triangle I=0.25 \mathrm{mag}$ in 30 minutes, then declined by 0.21 mag in 31 minutes. However, the shape of the light curve for standard stars is similar with that of BL Lac, with a amplitude of $0.14 \mathrm{mag}$ (see the bottom panel of Fig. 21). Thus, the microvariation in BL Lac is less convincing. The observations obtained on 08 November 1996 indicate that during the first hour the source was observed, it exhibited no significant variations. However, over the last 38 minutes the source underwent a rapid flare of $\triangle R=0.11 \mathrm{mag}$ (See Fig. 22). On 10 November, the source was 0.21 mag fainter (in the $R$ band) than it was at the end of the observation on 08 November. The source was at the same brightness level of the night before and exhibited no convincing microvariation 
during the observation on the night of 11 November (Fig. 23). Figure 24 presents the observation obtained on the night of 12 December 1996. Compared with the last observation obtained on 11 November, the source brightened about $0.5 \mathrm{mag}$ in the $V$ band, $0.47 \mathrm{mag}$ in the $R$ band and $0.39 \mathrm{mag}$ in the $I$ band. During the observation, the source exhibited no significant rapid variations on this night. The observations obtained on 14 December are displayed in Fig. 25. The light curve shows no significant variations, but compared with the observations obtained on the night before, the source faded by about $0.3 \mathrm{mag}$ in the $I$ band to the same brightness level with that in November 1996.

In general, the source exhibited no obvious long-term variation trends over the last two months of 1996. It kept at a faint level on which some irregular rapid variations were superimposed (see Figs. 21-25 and Table 2). Over the length of these observations, BL Lac had a total variability amplitude of $\triangle V=0.5 \mathrm{mag}, \triangle R=0.47 \mathrm{mag}, \triangle I=$ 0.39 mag. On JD $=2450398.090$ it declined to a minimum $B=16.62 \mathrm{mag}$, only $0.6 \mathrm{mag}$ brighter than the faintest level $B=17.2 \mathrm{mag}$ registered by Webb et al. (1988), and on JD $=2450399.068$ it declined to $V=$ $15.58 \mathrm{mag}$, which was $1.15 \mathrm{mag}$ brighter than the faintest level $V=16.73$ mag registered by Carini et al. (1992b).

\section{Conclusions}

We have presented some new results of CCD photometry for $7 \mathrm{BL}$ Lac objects. All of them exhibited microvariations during our observation. The XBL, H 0323+022, exhibited brightness oscillations on 15 December 1996 and 05 January 1997. The largest amplitude is about 0.63 mag. Another XBL, H 0548 - 322, exhibited smaller oscillation on 01 January 1997. Although in our observation the XBL H 2154 - 304 presented no brightness oscillation, there are reports about oscillation of about one day in this source (Smith et al. 1992; Courvoisier et al. 1995). During our observation, the four RBLs, PKS 0735+178, PKS 0754+101, OJ 287 and BL Lac did not show this kind of quick brightness oscillations or one-after-another flickers and then do not microvary very frequently. Carini et al. (1992b) have investigated microvariability in OJ 287 and BL Lac extensively. Their observation also recorded no oscillation of about one day or less, and microvariations in these two source do not appear very frequently. Dultzin-Hacyan, Takalo \& Benitez et al. (1997) have observed OJ 287 during a flare and found six microvariations on six nights out of ten nights. The largest amplitude is about $0.3 \mathrm{mag}$ and the microvariations do not appear very frequently. Microvariations in other RBLs, e.g. 0954+658 (Wagner et al. 1993), AP Librae (Carini et al. 1990a), OQ 530 (Carini et al. 1990b), 3C 66A (Takalo \& Sillanpää et al. 1996), AO 0235+164 (Rabbette et al. 1996), also do not appear very frequently. The only RBL that shows daily brightness oscillations is S5 $0716+714$
(Wagner et al. 1994). It thus seems that RBLs do not show microvariability more frequently than XBLs. If the relativistic jets in RBLs truly orient closer to the line of sight than the relativistic jets in XBLs do, then RBLs should have higher Doppler factors than XBLs, and those microvariations that are undetectable in XBLs should become detectable in RBLs. That is to say, RBLs should microvary more frequently than XBLs. However, variability behaviour in BL Lac objects is very complex, and even in a flare the microvariability behaviour during the onset of the flare and during the decline part of the flare is different (Dultzin-Hacyan et al. 1997). Therefore, it is necessary to carry out more photometric observations for BL Lac objects to further investigate whether RBLs do not microvary more frequently than XBLs.

Acknowledgements. We are grateful to National Science Foundation of China and ULOAC for their support of this work.

\section{References}

Begelman M.C., Blandford R.D., Rees M.J., 1984, Rev. Mod. Phys. 56, 255

Brindle C., et al., 1986, MNRAS 221, 739

Brinkman W., et al., 1994, A\&A 288, 433

Carini M.T., Miller H.R., 1992a, ApJ 385, 146

Carini M.T., Miller H.R., et al., 1990a, AJ 101, 1196

Carini M.T., Miller H.R., et al., 1992b, AJ 104, 15

Carini M.T., Miller H.R., et al., 1990b, AJ 100, 347

Courvoisier T., et al., 1995, sApJ 438, 108

Craine E.R., Warner J.W., 1973, ApJ 179, L153

Dultzin-Hacyan D., Takalo L.O., Benitez E., et al., 1997, Rev. Mex. A\&A 315, L13

Fanaroff B., Riley J.M., 1974, MNRAS 167, 31P

Ghisellini G., Maraschi L., 1989, ApJ 340, 181

Giommi P., et al., 1990, ApJ 356, 432

Heidt J., Wagner S.J., 1996, A\&A 305, 42

Jannuzi B.T., Smith P.S., Elston R., 1994, ApJ 428, 130

Jannuzi B.T., Smith P.S., Elston R., 1993, ApJS 85, 265

Kidger M., Takalo L.O., Sillanpää A., 1992, A\&A 264, 32

Kidger M., et al., 1995, A\&AS 113, 431

Massaro E., et al., 1995, A\&A 299, 339

Maraschi L., et al., 1986, ApJ 310, 325

Miller H.R., et al., 1976, AJ 81, 298

Miller H.R., AcAlister H.A., 1983, ApJ 272, 26

Miller H.R., Noble J.C., 1997, ASP Conf. Ser. 110, 17

Pica A.J., et al., 1980, AJ 85, 1442

Pica A.J., et al., 1988, AJ 96, 1215

Pollock J.T., et al., 1979, AJ 84, 1658

Rabbette M., et al., 1996, A\&A 310, 1

Shen B.S.P., Usher P.D., 1970, Nat 228, 1070

Sillanpää A., et al., 1988, ApJ 325, 628

Sillanpää A., Takalo, L.O., Pursimo, T., et al., 1996a, A\&A 305, L17

Sillanpää A., Takalo, L.O., Pursimo, T., et al., 1996b, A\&A 315, L13

Smith P.S., et al., 1985, AJ 90, 1184

Smith P.S., et al., 1991, ApJS 77, 67 
Smith P.S., et al., 1992, ApJ 400, 115

Stickel M., et al., 1991, ApJ 374, 431

Stoke J.T., et al., 1985, ApJ 298, 619

Takalo L.O., 1994, Vistas Astron. 38, 77

Takalo L.O., Sillanpää, A., et al., 1996, A\&AS 120, 313

Urry C.M., et al., 1993, ApJ 411, 611

Wagner S.J., et al., 1993, A\&A 271, 344

Wagner S.J., et al., 1994, AJ 111, 2187

Wagner S.J., Witzel A., 1995, ARA\&A 33, 163

Webb J.R., et al., 1988, AJ 95, 374

Weistrop D., 1973, Nat 241, 157
Xie G.Z., et al., 1987, A\&AS 67, 17

Xie G.Z., et al., 1988a, A\&AS 72, 163

Xie G.Z., et al., 1988b, AJ 96, 24

Xie G.Z., et al., 1989, A\&A 220, 89

Xie G.Z., et al., 1990, A\&A 229, 329

Xie G.Z., et al., 1991, A\&AS 87, 461

Xie G.Z., et al., 1992, ApJS 80, 683

Xie G.Z., et al., 1994, A\&AS 106, 361

Xie G.Z., et al., 1995, AJ 111, 1065

Zhang Y.H., Xie G.Z., 1996, A\&AS 116, 289 